\title{
Identification of pain-related psychological risk factors for the development and maintenance of pediatric chronic postsurgical pain
}

This article was published in the following Dove Press journal:

Journal of Pain Research

4 March 2013

Number of times this article has been viewed

\author{
M Gabrielle Pagé \\ Jennifer Stinson ${ }^{2,3}$ \\ Fiona Campbell ${ }^{2,4}$ \\ Lisa Isaac ${ }^{2,4}$ \\ Joel Katz ${ }^{1,4,5}$
}

'Department of Psychology, York University, ${ }^{2}$ Department of Anesthesia and Pain Medicine, Hospital for Sick Children, ${ }^{3}$ Lawrence S Bloomberg Faculty of Nursing, ${ }^{4}$ Department of Anesthesia, University of Toronto, ${ }^{5}$ Department of Psychology, Hospital for Sick Children, Toronto, ON, Canada
Correspondence: Joel Katz Department of Psychology, BSB 232, York University, 4700 Keele Street, Toronto ON M3J IP3, Canada Tel +I 4167362100 ext 40557 Email jkatz@yorku.ca
Background: The goals of this study were to examine the trajectory of pediatric chronic postsurgical pain (CPSP) over the first year after surgery and to identify acute postsurgical predictors of CPSP.

Methods: Eighty-three children aged 8-18 years (mean 13.8, standard deviation 2.4) who underwent major orthopedic or general surgery completed pain and pain-related psychological measures at 48-72 hours, 2 weeks (pain anxiety and pain measures only), and 6 and 12 months after surgery.

Results: Results showed that 1 year after surgery, $22 \%$ of children developed moderate to severe CPSP with minimal functional disability. Children who reported a Numeric Rating Scale pain-intensity score $\geq 3$ out of 10 two weeks after discharge were more than three times as likely to develop moderate/severe CPSP at 6 months and more than twice as likely to develop moderate/severe CPSP at 12 months than those who reported a Numeric Rating Scale pain score $<3$ (6-month relative risk 3.3, 95\% confidence interval 1.2-9.0 and 12-month relative risk $2.5,95 \%$ confidence interval $0.9-7.5)$. Pain unpleasantness predicted the transition from acute to moderate/severe CPSP, whereas anxiety sensitivity predicted the maintenance of moderate/ severe CPSP from 6 to 12 months after surgery.

Conclusions: This study highlights the prevalence of pediatric CPSP and the role played by psychological variables in its development/maintenance. Risk factors that are associated with the development of CPSP are different from those that maintain it.

Keywords: chronic postsurgical pain, children, adolescents, anxiety sensitivity

\section{Background}

Chronic postsurgical pain (CPSP) is defined as pain attributable to the surgical procedure and lasting for more than 2 months after surgery. ${ }^{1}$ The 1 -year prevalence of adult CPSP typically ranges between $1.5 \%$ and $10 \%$, although the prevalence can reach up to $70 \%$ in amputees and after thoracotomy. ${ }^{2}$ CPSP may be accompanied by moderate to severe pain intensity and pain-related disability. ${ }^{3}$ The variability in prevalence rates is due not only to the nature of the surgical procedure and time since surgery but also to the criteria used to classify patients: rates are typically higher in studies that include any report of pain (ie, regardless of intensity) and lower when an upper limit on intensity is specified.

In contrast to the adult literature, there are not a lot of data on CPSP in children and/or adolescents. Moreover, the issue of how best to define pediatric CPSP has not been addressed. To date, only retrospective studies of pediatric CPSP have been reported. These studies estimate the prevalence of CPSP to be between $13 \%$ more than 
15 years after surgery to $50 \% 3$ months after surgery. ${ }^{4-7}$ Pain intensity reported in these studies also ranged from mild to severe. Retrospective studies are subject to recall bias and do not permit identification of risk factors for the development of CPSP.

Biopsychosocial models of chronic pain highlight the important role played by biomedical, social/environmental, and psychological factors in the development and maintenance of CPSP in various surgical populations. ${ }^{8-10}$ Recent reviews of adult CPSP have identified different categories of risk factors, including patient-related (eg, previous or concurrent pain experience, acute postsurgical pain [APSP] intensity, younger age, female sex, analgesic consumption during the first week after surgery ${ }^{11-13}$ ), social/environmental (eg, social support $\left.{ }^{8}\right)$, and psychological (eg, anxiety, pain catastrophizing, surgery-related fears, psychological vulnerabilities, emotional numbing). ${ }^{11,12,14-16}$

Factors associated with the development and maintenance of CPSP have yet to be examined in pediatric populations. ${ }^{17}$ The focus of the present study is on patient-related factors, including age, sex, acute pain intensity, and unpleasantness (how horrible, yucky, or unpleasantness the feeling of pain is), and analgesic consumption, as well as pain-related psychological factors, including pain anxiety, pain catastrophizing, and anxiety sensitivity. Pain anxiety refers to cognitive, emotional, physiological, and behavioral reactions that one exhibits in association to an actual or anticipated pain experience. ${ }^{18,19}$ Pain catastrophizing refers to the rumination, feeling of helplessness, and magnification of the experience of pain. ${ }^{20,21}$ Anxiety sensitivity refers to the degree to which one interprets anxiety-related symptoms as being associated with potentially harmful somatic, psychological, or social consequences..$^{22}$ Research has shown that these pain-related psychological constructs are associated with pain severity and disability in community and clinical pediatric populations ${ }^{23-27}$ and are important factors in empirically validated models of chronic pain, including the diathesis-stress model of chronic pain and disability ${ }^{28}$ and the cognitive-behavioral fear-avoidance model of chronic pain. ${ }^{29-31}$ However, the role of these constructs in the development and maintenance of pediatric CPSP has yet to be examined.

Findings from the adult literature indicate that the factors predicting the transition to chronicity (ie, from the acute postoperative phase to $\sim 6$ months after surgery) are different from those involved in the maintenance of CPSP (ie, from 6 months onward), ${ }^{32,33}$ and this has also been found in at least one pediatric study of patients with widespread body pain. ${ }^{34}$ These findings point to the importance of examining in children the factors that predict the transition to chronicity separately from those that predict its persistence, since different mechanisms and risk factors might be involved at each stage.$^{33}$ They also highlight the importance of assessing outcomes at more than one time point after surgery, ${ }^{35}$ since the transition to CPSP and related disability is a dynamic process that evolves over time. ${ }^{33}$

The goals of this study were to (1) describe the course of pediatric CPSP by modeling pain intensity and pain unpleasantness trajectories over the first year after surgery, (2) identify risk factors for the transition from APSP to CPSP (ie, development of CPSP), and (3) identify risk factors for the maintenance of CPSP. It is hypothesized that (1) CPSP of moderate to severe intensity will have a prevalence rate similar to the adult literature $(\sim 10 \%)$, (2) pain anxiety, pain catastrophizing, and anxiety sensitivity will predict the development of CPSP, and (3) pain anxiety, pain catastrophizing, and anxiety sensitivity will predict the maintenance of CPSP. APSP was measured 48-72 hours and approximately 2 weeks after surgery, and CPSP was measured 6 and 12 months after surgery. Children were classified as having moderate/severe CPSP if they reported a Numeric Rating Scale (NRS) for pain intensity score $\geq 4^{36,37} 6$ and/or 12 months after surgery; otherwise, they were classified as not having or having mild CPSP.

\section{Materials and methods Participants and recruitment}

Children between the ages of 8 and 18 years undergoing either orthopedic (ie, scoliosis, osteotomy, plate insertion tibial/ femur, open hip reduction, hip capsulorrhaphy) or general surgical (ie, thoracotomy, thoracoabdominal surgery, Nuss or Ravitch surgical procedure to correct pectus excavatum, sternotomy, laparotomy) procedures were eligible to participate in this study. Surgical procedures were selected on the basis of expected postoperative pain. The authors chose procedures that are known to produce moderate to severe pain while in hospital. This was done to maximize the clinical relevance of the study results, and for reasons of sample size to maximize the proportion of patients expected to develop CPSP under the hypothesis that acute pain intensity impacts the development of chronic pain. Although there is variability in recovery time, depending on the procedure, it is generally known that acute pain improves over time after spinal fusion for scoliosis ${ }^{38}$ (the usual indication for spinal fusion in this age-group).

Children were excluded if they had a developmental or cognitive delay, had cancer, or were not fluent in written and/ or spoken English. 


\section{Questionnaires}

\section{Child Pain Anxiety Symptoms Scale}

The Child Pain Anxiety Symptoms Scale (CPASS) ${ }^{39}$ is a 20 -item scale for children adapted from the adult PASS-20. ${ }^{40}$ For each statement, children are asked to rate the extent to which they think, act, or feel that way on a scale from 0 (never think, act, or feel that way) to 5 (always think, act, or feel that way). Total scores range from 0 to 100, with higher scores indicating higher levels of pain anxiety. The CPASS is composed of four subscales: cognitive, escape/avoidance, fear, and physiological anxiety. The CPASS showed excellent internal consistency $(\alpha=0.90)$ in a community sample of children, ${ }^{39}$ as well as in the present study $(\alpha=0.92-0.96) .{ }^{41}$ In addition, the CPASS correlated more strongly with pain catastrophizing $(r=0.63)$ and anxiety sensitivity $(r=0.60)$ than with general anxiety $(r=0.44)$ (suggesting adequate construct validity), and was significantly associated with how often children reported pain. ${ }^{39}$

\section{Childhood Anxiety Sensitivity Index}

The Childhood Anxiety Sensitivity Index (CASI) ${ }^{42}$ assesses the extent to which the respondent interprets anxiety-related symptoms (eg, increased heart rate, feeling nauseated) as indicators of potentially harmful somatic, psychological, and/or social consequences. ${ }^{22}$ The scale is composed of 18 items, such as "It scares me when my heart beats fast" and "It scares me when I feel like I'm going to throw up." Items are rated on a scale ranging from 1 (none) to 3 (a lot). Total scores range from 18 to 54, with higher scores indicating higher levels of anxiety sensitivity. The CASI has good internal consistency $(\alpha=0.87)$ and test-retest reliability $(r=0.76)$, as well as adequate convergent and discriminant validity. ${ }^{42}$ Internal consistency for the present study was excellent $(\alpha=0.87-0.93)$.

\section{Pain Catastrophizing Scale - Children}

The Pain Catastrophizing Scale - Children (PCS-C) $)^{43}$ is a 13-item self-report measure assessing the extent to which children worry, amplify, and feel helpless about their current or anticipated pain experience. ${ }^{43}$ The scale was modified for use with children based on the adult PCS. ${ }^{44,45}$ For each item, children are asked to rate, on a scale from 0 (not at all) to 4 (extremely), how strongly they experience a particular thought when they have pain. Total scores range from 0 to 52 , with higher scores indicating higher levels of pain catastrophizing. The PCS-C comprises three subscales: rumination, magnification, and helplessness.
Preliminary results suggest that the PCS-C has good internal consistency $(\alpha=0.90)$ and correlates highly with pain intensity $(r=0.49)$ and disability $(r=0.50) .{ }^{43}$ Internal consistency for the present study was excellent $(\alpha=0.93)$.

\section{Functional Disability Inventory}

The Functional Disability Inventory (FDI ${ }^{46}$ is a 15 -item scale that assesses the extent to which children experience difficulties in completing specific tasks (eg, walking to the bathroom, eating regular meals, and being at school all day). Typically, the FDI is used as a 5-point Likert scale and yields total scores ranging from 0 to 60 . Inadvertently, the FDI in the present study was measured using a 4-point Likert scale and omitted the original 2 (some trouble). Children in this study rated each item on a scale from 0 to 3 : ( 0 , no trouble; 1 , a little trouble; 2 , a lot of trouble; and 3, impossible), yielding total scores ranging from 0 to 45 . The FDI has excellent internal consistency $(\alpha=0.86-0.91)$ and good test-retest reliability at 2 weeks $(r=0.74)$ and 3 months $(r=0.48)$. The FDI has been used with many pediatric populations, including children with chronic pain ${ }^{47-49}$ and postsurgical pain. ${ }^{50}$ Internal consistency for the present study was excellent $(\alpha=0.83-0.89)$.

\section{Numeric Rating Scale for pain intensity (NRSI) and pain unpleasantness (NRSU)}

The NRS is a verbally administered scale that measures pain intensity ("How much pain do you feel right now?"). The NRS was also used to measure pain unpleasantness ("How unpleasant/horrible/yucky is the pain right now?"). The end points represent the extremes of the pain experience. Since there are no agreed-upon NRS anchors for measuring pain in children and adolescents, ${ }^{51}$ the following anchors were used in the present study: for pain intensity, $0=$ no pain at all to $10=$ worst possible pain; for pain unpleasantness, $0=$ not at all unpleasant/horrible/yucky to $10=$ most unpleasant/horrible/yucky feeling possible. The NRS for pain intensity has been validated as an APSP measure in children aged 7-17 years; it correlated highly with the Visual Analog Scale $(r=0.89)$ and the revised Faces Pain Scale $(r=0.87) .{ }^{52}$

\section{Preoperative pain experience}

Retrospectively (48-72 hours after surgery), children were asked to answer the following question: "Before surgery, how much pain did you have in general?" using one of four verbal descriptors: 0 , no pain; 1 , a little bit of pain; 2 , a medium amount of pain; or 3, a lot of pain. 


\section{Hospital chart review}

The following information was collected from children's hospital charts: preoperative information (eg, ongoing pain problems, regular pain medications, previous surgery), perioperative information (eg, type of procedure, length of surgery), and postoperative information (eg, pain medication on the first day after surgery).

\section{CPSP questionnaire}

This questionnaire was designed to evaluate children's pain experience postoperatively and included the following questions: "Do you ever feel pain in the area of your body where the surgery was done?" "If yes, how often did you feel pain?" "On average, how much pain do you feel on a scale from 0 to 10 ?" "When you feel pain, where exactly is the pain you are usually feeling?" "What kind of pain do you usually feel?" "Are you taking any medication for your pain?" "Are you using any alternative method to relieve your pain?" "Are you seeing a doctor specifically for your pain?"

We also administered the Center for Epidemiological Studies Depression Scale for Children ${ }^{53}$ and the Multidimensional Anxiety Scale for Children ${ }^{54}$ at $24-48$ hrs, 6 months, and 12 months after surgery, but do not report these data here.

\section{Procedure}

The study was reviewed and approved by the research ethics boards of the Hospital for Sick Children and York University. Nurses who were not part of the research team approached potential participants to ask whether they would be interested in learning about the study. Children and one of their parents (who had expressed an interest in the study) were then approached by a research team member 48-72 hours after surgery. After obtaining informed written parental consent and consent or assent from children, a research team member read to the child the following questionnaires and recorded their responses to each item: CPASS, PCS-C, CASI, NRSI, NRSU, and preoperative experience. The order of administration of questionnaires was randomized (http://www.randomization.com) within participants to minimize potential order and fatigue effects. Telephone follow-ups were conducted approximately 2 weeks (CPASS, FDI, NRSI, and NRSU) and 6 and 12 months (CPASS, PCS-C, CASI, NRSI, NRSU, FDI, and CPSP questionnaire) after surgery by a research assistant who verbally administered the questionnaires to the children. A verbal mode of administration of questionnaires was chosen because some participants could not move their arms or sit up 48-72 hours after surgery and thus could not complete the questionnaires themselves. Verbally administering the questionnaires to all participants minimized response bias due to different types of administration of questionnaires. In addition, follow-ups were conducted over the telephone, and as such verbally administering the questionnaires 48-72 hours after surgery ensured that the same method of administration was preserved throughout the study.

Parents also completed measures; these results will be presented in a separate manuscript.

\section{Data analysis}

Data was screened for univariate outliers on pain-related psychological predictor variables (pain anxiety, pain catastrophizing, and anxiety sensitivity), multivariate outliers (squared Mahalanobis distance as a probability $\left[\chi^{2}\right]<0.001$ ), as well as skewness and kurtosis.

Outlier analysis revealed that none of the data points was both a univariate and a multivariate outlier; as such, all participants were retained for the analyses. Visual examination of the predictor variable plots as well as skewness and kurtosis significance testing did not reveal the presence of nonnormality. Skewness and kurtosis significance testing (estimate/standard error $>3$ ) did reveal, however, nonnormality of two outcome variables: pain intensity and pain unpleasantness at 2 weeks, 6 months, and 12 months after surgery. A Poisson distribution best represents the variability of the outcome variables, given that at all three time points, approximately half of the sample did not report experiencing CPSP on the NRSI and NRSU, whereas scores for the other half of the participants ranged between 1 and 10, with a greater proportion reporting lower levels of pain. Nonnormality of the outcome variables was addressed either through square-root transformation (NRSI[2] ${ }^{\mathrm{t}}, \mathrm{NRSU}[2]^{\mathrm{t}}, \mathrm{NRSI}[6]^{\mathrm{t}}, \mathrm{NRSU}[6]^{\mathrm{t}}, \mathrm{NRSI}[12]^{\mathrm{t}}$, and NRSU[12] $\left.{ }^{t}\right)$ or by using a Poisson (loglinear) distribution in the analyses whenever possible.

\section{Description of moderate/severe pediatric CPSP and modeling of pain intensity and pain unpleasantness trajectories}

The Pearson Chi-squared test was used to examine the presence of sex differences in children's chronic pain status (moderate/severe CPSP or no/mild CPSP [moderate to severe CPSP is defined as NRS $\geq 4$ ]) 6 and 12 months after surgery.

\section{CPSP intensity and unpleasantness trajectories across time}

A generalized estimating equation (GEE) using Poisson log-linear distribution was used to compare the pain 
intensity (model 1) and pain unpleasantness (model 2) trajectories (pattern of pain scores over time) of children with moderate/severe CPSP to those with no/mild CPSP. In addition to the linear time variable, time was also transformed to generate an asymptotic curve to model the decline of pain scores over time. This asymptotic function better characterizes the pattern by which pain scores decrease over time; namely, a more rapid decline in pain over the first days/weeks and a slower decline from 2 weeks to 6 and 12 months after surgery. The following variables (and their interactions) were sequentially entered in each model and retained when statistically significant: time (in months, with 48-72 hours as the starting point - zero), asymptotic time (time transformed), previous surgical experience, and pain status (moderate/severe CPSP vs no/ mild CPSP).

\section{Risk factors for the transition from APSP to CPSP}

Binary logistic regression analyses were used to examine if sex, age, analgesic consumption of day 1 after surgery, and 48- to 72-hour pain intensity, pain unpleasantness, pain anxiety, pain catastrophizing, and anxiety sensitivity predicted the development of moderate/severe CPSP 6 months (model 1) and 12 months (model 2) after surgery. Predictors were entered in the models using a forward conditional method.

\section{Risk factors for the maintenance of moderate/ severe CPSP}

Binary logistic regression analysis was used to examine if age, sex, and 6-month (model 2) pain anxiety (CPASS), pain catastrophizing (PCSC), anxiety sensitivity (CASI), pain intensity (NRSI), and pain unpleasantness (NRSU) predicted the maintenance of moderate/severe CPSP 12 months after surgery. Predictors were entered in the model using a forward conditional method. All statistical analyses were conducted using SPSS v.19 (2010, IBM Corporation, Armonk, NY, USA).

\section{Sample-size estimation}

Sample size was estimated a priori using $G^{*}$ Power version 3.1 (Franz Faul, Universitat Kiel, Germany). ${ }^{55}$

\section{Description of pediatric moderate/severe CPSP and modeling of pain intensity and pain unpleasantness trajectories}

Sample-size estimation for the Pearson Chi-squared test showed that a sample size of 50 participants would be required to detect a medium to large effect size $(w=0.40)$ with $\alpha=0.05$, power $=80 \%$, and degrees of freedom $=1$. Sample-size estimation for the mixed-effects models, including GEE and generalized linear mixed-effects models, is difficult to perform, given the multiple combinations of mixed and random effects involved. However, it has been shown that sample size required for a mixed-effects model is comparable to the sample size required for linear regression analysis for balanced designs. ${ }^{56}$ For a multiple regression analysis with four predictors (time, asymptotic time, prior surgical procedure, and pain status), 65 participants would be required for $\alpha=0.05$, power $=80 \%$, and a medium to large effect size $\left(f^{2}=0.25\right)$.

\section{Risk factor analyses of the transition from APSP to CPSP and maintenance of moderate/severe CPSP}

Sample-size analysis showed that 60 participants would be required for a multiple logistic regression analysis Hsieh's test procedure, ${ }^{57}$ with odds ratio $=2.5$, probability $(y=1 \mid x=1) \mathrm{H}_{0}=0.5, \alpha=0.017, R^{2}$ other $x=0.16$, and power $=80 \%$.

Taking into account an attrition rate of $\sim 30 \%$ due to dropouts and losing patients to follow-up, we recruited 83 patients to ensure we would have sufficient power for our analyses at the various time points after surgery.

\section{Results}

\section{Recruitment}

Recruitment took place between July 2008 and September 2010. Details of the recruitment process are presented in Figure 1. Eighty-three children/parents provided assent/ consent to participate, of whom $69(83 \%)$ also completed the telephone follow-up approximately 2 weeks after discharge from hospital ( mean $=15.6$ days, standard deviation [SD] $=2.15)$. Sixty-one $(73 \%)$ and $59(71 \%)$ participants completed the 6- and 12-month follow-ups, respectively.

There were no significant differences between children who completed vs those who did not complete the 6-month follow-up on measures of pain intensity $(P=0.087)$, pain unpleasantness $(P=0.347)$, pain anxiety $(P=0.741)$, pain catastrophizing $(P=0.864)$, anxiety sensitivity $(P=0.363)$ measured 48-72 hours after surgery, analgesic consumption on day $1(P=0.335)$, age $(P=0.920)$, or sex $(P=0.934)$. There were no significant differences between children who completed vs those who did not complete the 12-month follow-up on measures of pain intensity $(P=0.412)$, pain unpleasantness $(P=0.090)$, pain anxiety $(P=0.668)$, pain catastrophizing $(P=0.555)$, anxiety sensitivity $(P=0.601)$ measured $48-72$ hours after 


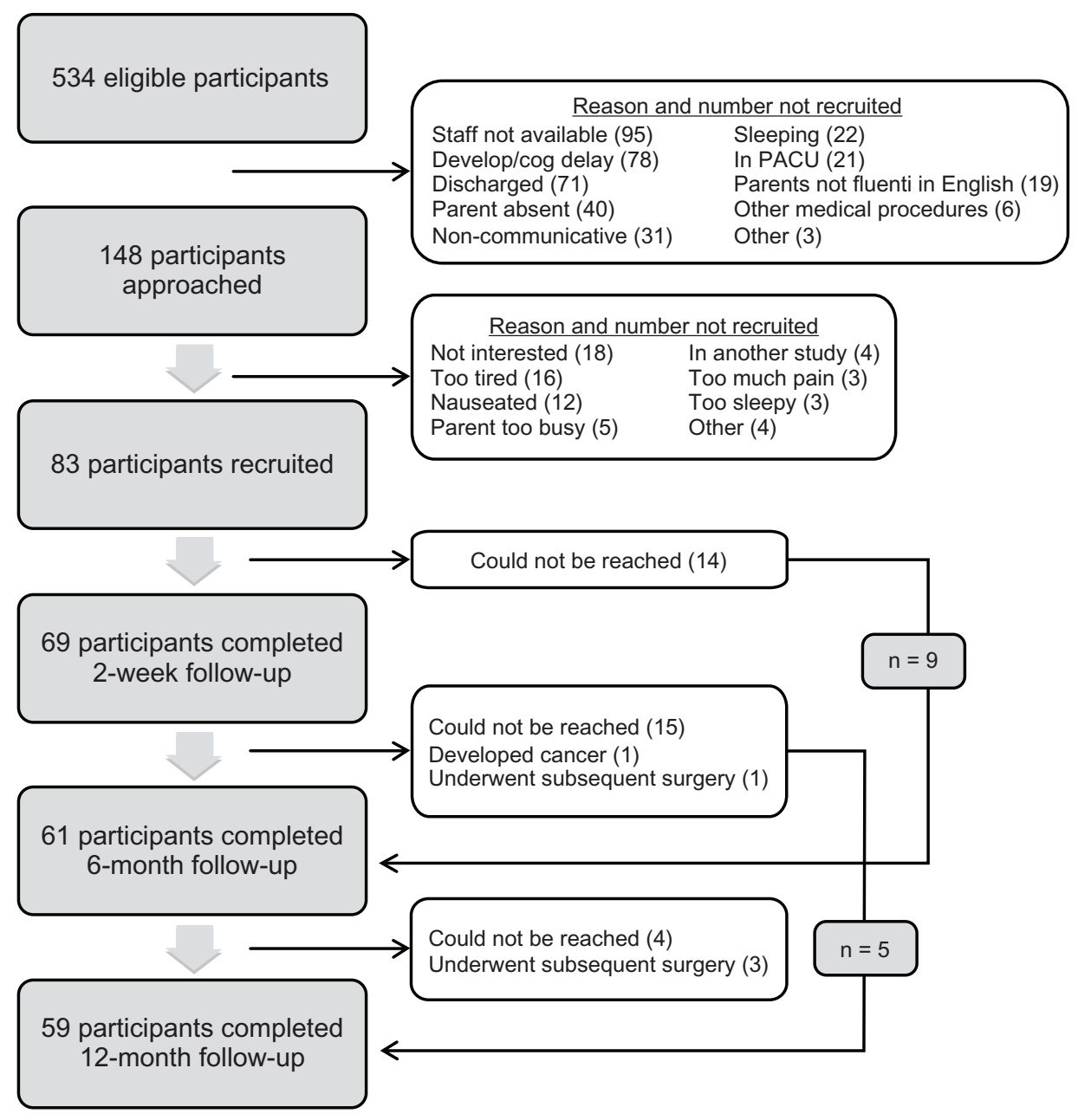

Figure I Details of recruitment process.

Abbreviation: PACU, post-anesthesia care unit.

surgery, analgesic consumption on day $1(P=0.255)$, age $(P=0.572)$ or $\operatorname{sex}(P=0.677)$.

\section{Descriptive statistics}

The final sample comprised 83 children (female $=56$ [67.5\%]) aged between 8 and 18 years (mean age in years for total sample $=13.8, \mathrm{SD}=2.4$; girls $=14.0, \mathrm{SD}=2.3$; boys $=13.5, \mathrm{SD}=2.6$ ). The majority of children (or parents) in the sample self-identified as Caucasian $(\mathrm{n}=53,64 \%) ; 12 \%$ self-identified as Asian, $8.4 \%$ as African-Caribbean/AfricanCanadian, $4.8 \%$ as Middle Eastern, 3.6\% as Hispanic, and $7.2 \%$ were categorized as "Other." Eighty-nine percent of children spoke English as their first language at home. The majority of children underwent surgery for scoliosis (spinal fusion) $(\mathrm{n}=42,50.6 \%)$ or osteotomy $(\mathrm{n}=25,30.1 \%)$; eight children $(9.6 \%)$ underwent Nuss $(\mathrm{n}=5)$ or Ravitch $(\mathrm{n}=3)$ procedures, seven children $(8.4 \%)$ had a laparotomy, and one child had a thoracotomy. The present surgery was the first for 44 children (53\%); 39 others had previously undergone other surgical procedures (mean $=2.0, \mathrm{SD}=1.6$, range $=1-7)$. When asked to rate the level of presurgical pain they had experienced, the majority of children (80.7\%) reported no pain or a little bit of pain. Two children who developed CPSP at 6 months and one child who developed CPSP at 12 months had reported moderate to severe pain before surgery. Eighteen out of 20 children with baseline pain underwent surgery for scoliosis or osteotomy, which corrected the source of their baseline pain.

Significant differences were not found between moderate/ severe CPSP and no/mild CPSP at $6\left(\chi^{2}=3.61, P=0.306\right)$ or 12 months $\left(\chi^{2}=1.91, P \leq 0.592\right)$ after surgery across the different types of surgical procedures. Mean \pm SD of morphine equivalent analgesic consumption on the first day following surgery was $1.21 \pm 1.02 \mathrm{mg} / \mathrm{kg}(62.81 \pm 57.6 \mathrm{mg})$. Mean values 
Table I Means (standard deviations) of pain and related psychological variables for boys, girls, and the total sample measured 6 and 12 months after surgery

\begin{tabular}{|c|c|c|c|c|c|c|}
\hline & \multicolumn{3}{|c|}{$\begin{array}{l}6 \text { months after surgery } \\
(n=61)\end{array}$} & \multicolumn{3}{|c|}{$\begin{array}{l}\text { I } 2 \text { months after surgery } \\
(\mathrm{n}=59)\end{array}$} \\
\hline & Boys & Girls & Total & Boys & Girls & Total \\
\hline $\begin{array}{l}\text { Pain anxiety } \\
\text { (CPASS) }\end{array}$ & $29.4(21.2)$ & $36.9(18.6)$ & $34.4(19.6)$ & $20.1(17.9)$ & $32.4(18.3)$ & $28.4(18.9)^{\dagger}$ \\
\hline $\begin{array}{l}\text { Pain catastrophizing } \\
\text { (PCSC) }\end{array}$ & $10.7(13.4)$ & $16.0(9.9)$ & | $4.3(|| .3)$ & $8.9(7.4)$ & $14.0 \mid(10.0)$ & $12.4(9.5)$ \\
\hline \multirow{2}{*}{$\begin{array}{l}\text { Anxiety sensitivity } \\
\text { (CASI) }\end{array}$} & $30.3(7.4)$ & $33.2(6.6)$ & $32.3(6.9)$ & $26.2(6.7)$ & $31.4(7.8)$ & $29.7(7.8)^{\dagger}$ \\
\hline & (n) & (n) & (n) & (n) & (n) & (n) \\
\hline \multicolumn{7}{|l|}{ Pain } \\
\hline Moderate/severe CPSP & 3 & II & 14 & 2 & II & 13 \\
\hline No/mild CPSP & 17 & 30 & 47 & 18 & 28 & 46 \\
\hline \multicolumn{7}{|l|}{ Pain intensity (NRSI) } \\
\hline Moderate/severe CPSP & $6.7(3.1)$ & $5.0(\mathrm{I} . \mathrm{I})$ & $5.4(\mathrm{I} .7)$ & $6.0(1.4)$ & $4.5(0.8)$ & $4.8(1.0)$ \\
\hline No/mild CPSP & $1.4(1.2)$ & $0.6(1.1)$ & $0.9(1.2)^{\dagger}$ & $0.4(0.9)$ & $0.9(1.2)$ & $0.7(I .1)$ \\
\hline \multicolumn{7}{|c|}{ Pain unpleasantness (NRSU) } \\
\hline Moderate/severe CPSP & $5.3(4.0)$ & $4.6(1.8)$ & $4.8(2.3)$ & $6.5(0.7)$ & $4.2(1.2)$ & $4.5(1.4)^{\dagger}$ \\
\hline No/mild CPSP & $2.6(1.9)$ & $2.1(1.0)$ & $2.4(1.5)$ & $3.0(1.0)$ & $2.6(1.2)$ & $2.7(1.1)$ \\
\hline \multicolumn{7}{|c|}{ Functional disability (FDI) } \\
\hline Moderate/severe CPSP & $10.0(9.8)$ & $6.5(5.3)$ & $7.2(6.2)$ & $5.0(1.4)$ & $6.5(4.3)$ & $6.3(4.0)$ \\
\hline No/mild CPSP & $5.2(6.0)$ & $6.7(4.8)$ & $6.2(5.2)$ & $1.7(2.3)$ & $3.4(3.8)$ & $2.7(3.4)$ \\
\hline
\end{tabular}

Note: †Significant sex differences $(P<0.05)$.

Abbreviations: CPSP, chronic postsurgical pain; CPASS, Child Pain Anxiety Symptoms Scale; PCSC, Pain Catastrophizing Scale - Children; CASI, Childhood Anxiety Sensitivity Index; NRSI, Numeric Rating Scale for pain intensity; NRSU, Numeric Rating Scale for pain unpleasantness; FDI, Functional Disability Inventory (measured on a scale from 0 to 3$)$.

for boys and girls on the relevant psychological and pain measures at 6 and 12 months after surgery are presented in Table 1.

\section{Description of moderate/severe pediatric CPSP and modeling of pain intensity and pain unpleasantness trajectories}

Incidence and frequency of moderate/severe CPSP (NRSI $\geq 4)$ measured 6 and 12 months after surgery are presented in Table 2. Almost one-quarter of children reported experiencing moderate to severe pain at the surgical site at $6(n=14$ out of a total of $61,23 \%)$ and $12(n=13$ out of a total of $59,22 \%)$ months after surgery. Four children had CPSP at both 6 and 12 months. Three children who had pain at 6 months did not complete the 12-month follow-up, and as such their CPSP status at 12 months is unknown. Similarly, three children had CPSP at 12 months but did not complete the 6 month follow-up. Seven children with CPSP at 6 months no longer had pain at the 12-month follow-up. Lastly, six children who did not have CPSP at 6 months subsequently reported it at 12 months.

There were no significant sex differences in the presence/absence of moderate to severe CPSP $6(P=0.353)$ or $12(P=0.184)$ months after surgery. Six months after surgery, more than one-third of children $(n=5)$ with moderate/severe CPSP reported experiencing pain at least once a day, while only one child reported similar pain frequency 12 months after surgery. Average pain intensity and pain unpleasantness remained relatively constant over time. Distribution of pain intensity and pain unpleasantness scores for children with moderate/severe CPSP is presented in Figure 2. Moderate to severe CPSP was accompanied by low levels of functional disability at both $6($ mean $=7.2, \mathrm{SD}=6.2)$ and 12 months $($ mean $=6.3$, $\mathrm{SD}=4.0)$.

\section{Pain intensity trajectory}

GEE using Poisson log-linear distribution was used to examine differences in pain intensity over time between children with no or mild CPSP versus children who developed moderate or severe CPSP (at 6 and/or 12 months after surgery). The final model revealed a significant interaction between pain status and asymptotic time (Wald $\chi^{2}=38.9, P<0.001$ ) (see Table 3 and Figure 3 ) in predicting pain intensity scores. That is, pain intensity scores declined nonlinearly and at a more rapid rate in the no/mild CPSP group compared to the moderate/severe CPSP group. 
Table 2 Incidence and characteristics of pediatric CPSP 6 and 12 months after major orthopedic or general surgery

\begin{tabular}{|c|c|c|}
\hline & $\begin{array}{l}6 \text { months after } \\
\text { surgery } \\
\text { n (\%) }\end{array}$ & $\begin{array}{l}\text { I } 2 \text { months after } \\
\text { surgery } \\
n(\%)\end{array}$ \\
\hline No/mild CPSP ${ }^{\dagger}$ & $n=47(77 \%)$ & $n=46(78 \%)$ \\
\hline \multicolumn{3}{|c|}{ Time for pain to subside, $n$ (\%) } \\
\hline Within a few days & $3(6.3)$ & $3(6.5)$ \\
\hline Within a week & $8(17.0)$ & $\mathrm{I}(2.2)$ \\
\hline I-6 months & $17(36.3)$ & $22(47.8)$ \\
\hline $6-12$ months & $\mathrm{N} / \mathrm{A}$ & $5(10.9)$ \\
\hline $\begin{array}{l}\text { Still experiencing pain } \\
(N R S \leq 3)\end{array}$ & $19(40.4)$ & $15(32.6)$ \\
\hline Moderate/severe CPSP ${ }^{\dagger}$ & $n=14(23 \%)$ & $n=13(22 \%)$ \\
\hline \multicolumn{3}{|l|}{ Pain frequency, n (\%) } \\
\hline$<$ once a month & & $2(15.4)$ \\
\hline $\mathrm{I}-2$ per month & $3(2 \mid .4)$ & $3(23.1)$ \\
\hline Once a week & $3(2 \mid .4)$ & $4(30.8)$ \\
\hline >once a week & $3(2 \mid .4)$ & $3(23.1)$ \\
\hline Once a day & I (7.I) & \\
\hline$>$ once a day & $3(2 \mid .4)$ & I (7.7) \\
\hline Constant & $\mathrm{I}(7.1)$ & \\
\hline \multicolumn{3}{|l|}{ Pain location, n (\%)* } \\
\hline Scar/incision & $5(35.7)$ & $2(15.4)$ \\
\hline \multicolumn{3}{|l|}{ Skin } \\
\hline Muscle/deep tissue & $10(71.4)$ & $4(30.8)$ \\
\hline Other & $2(14.3)$ & I (7.7) \\
\hline \multicolumn{3}{|l|}{ Description of pain, $\mathbf{n}(\%)^{*}$} \\
\hline Aching & $5(35.7)$ & $6(46.2)$ \\
\hline Burning/hot & I (7.I) & \\
\hline Cramping/squeezing & $4(28.6)$ & $6(46.2)$ \\
\hline Shooting & $7(50.0)$ & I (7.7) \\
\hline Stabbing & $3(2 \mid .4)$ & I (7.7) \\
\hline Tingling & $2(14.3)$ & \\
\hline Sharp & $4(28.6)$ & I (7.7) \\
\hline Dull & & $2(15.4)$ \\
\hline Throbbing & & $4(30.8)$ \\
\hline Stiff/sore & $3(2 \mid .4)$ & $2(15.4)$ \\
\hline Other & & $3(23.1)$ \\
\hline \multicolumn{3}{|l|}{ Taking medication, $\mathbf{n}(\%)$} \\
\hline Yes & $8(57)$ & $\mathrm{I}(7.7)$ \\
\hline No & $6(43)$ & $12(92.3)$ \\
\hline \multicolumn{3}{|c|}{ Seeing doctor for pain, $\mathbf{n}(\%)$} \\
\hline Yes & $6(43)$ & $2(15.4)$ \\
\hline No & $8(57)$ & II (84.6) \\
\hline \multicolumn{3}{|c|}{ Using alternative method to relieve pain, $\mathbf{n}(\%)$} \\
\hline Yes & $6(43)$ & $4(30.8)$ \\
\hline No & $8(57)$ & $9(69.2)$ \\
\hline
\end{tabular}

Notes: ${ }^{\dagger}$ Among children who completed both the 6- and 12-month follow-ups, six children who did not report pain 6 months after surgery reported pain at the surgical site at the 12-month follow-up. Seven children who reported pain at the surgical site 6 months after surgery stated that they no longer experienced pain at the 12-month follow-up. The remaining children either reported pain at the surgical site at both the 6- and 12-month follow-ups $(n=4)$ or no pain at either of these two time points $(n=38)$; *some participants identified more than one pain location and description of pain.

Abbreviations: CPSP, chronic postsurgical pain; NRS, numeric rating scale; N/A, not applicable.
Based on the results of the GEE model (see Figure 3) a cutoff score of 3.0 on the NRSI 2 weeks after surgery separated children who reported moderate/severe CPSP from those who reported no/mild CPSP. Table 4 shows the relative risks and $95 \%$ confidence intervals (CIs) for developing moderate/severe vs no/mild CPSP at 6 and 12 months after surgery, using a cutoff score of 3.0 on the NRSI and NRSU at 2 weeks after discharge from hospital. Children who reported higher pain intensity (NRSI $\geq 3.0) 2$ weeks after discharge were 3.3 (95\% CI 1.2-9.0) times more likely to report moderate/severe CPSP 6 months after surgery than children who reported lower pain intensity (NRSI < 3.0) and 0.7 (95\% CI 0.4-1.1) times less likely to be pain-free or report mild pain intensity 6 months after surgery. Likewise, children who reported higher pain intensity (NRSI $\geq 3.0$ ) 2 weeks after discharge were 2.5 (95\% CI 0.9-7.5) times more likely to report moderate/severe CPSP 12 months after surgery as children who reported lower pain intensity (NRSI < 3.0) and $0.8(95 \% \mathrm{CI} 0.5-1.1)$ times less likely to be pain-free or report mild pain intensity 12 months after surgery.

\section{Pain unpleasantness trajectory}

A similar model was run to examine the pain unpleasantness trajectory of children with moderate/severe CPSP versus children with no/mild CPSP. The final model revealed a significant interaction between pain status and asymptotic time (Wald $\chi^{2}=21.4, P<0.001$ ) (see Table 3 and Figure 3 ) in predicting pain unpleasantness scores. That is, pain unpleasantness scores declined nonlinearly and pain unpleasantness scores declined faster in the no/mild CPSP group compared to the moderate/ severe CPSP group.

Table 4 shows the risk of developing moderate/severe CPSP vs no/mild CPSP at 6 and 12 months after surgery, using a cutoff score of 3.0 on the NRSU at 2 weeks. Also shown are the relative risks and $95 \%$ CIs. Children who reported higher pain unpleasantness (NRSU $\geq 3.0$ ) 2 weeks after discharge were 2.5 times more likely to report moderate/severe CPSP 6 months after surgery than children who reported lower pain unpleasantness (NRSI $<3.0$ ), and 0.8 times less likely to be pain-free or report mild CPSP 6 months after surgery. Likewise, children who reported higher pain unpleasantness (NRSI $\geq 3.0) 2$ weeks after discharge were 2.7 times more likely to report moderate/severe CPSP 12 months after surgery as children who reported lower pain unpleasantness (NRSI $<3.0$ ), and 0.8 times less likely to be pain-free or report mild CPSP 12 months after surgery. 

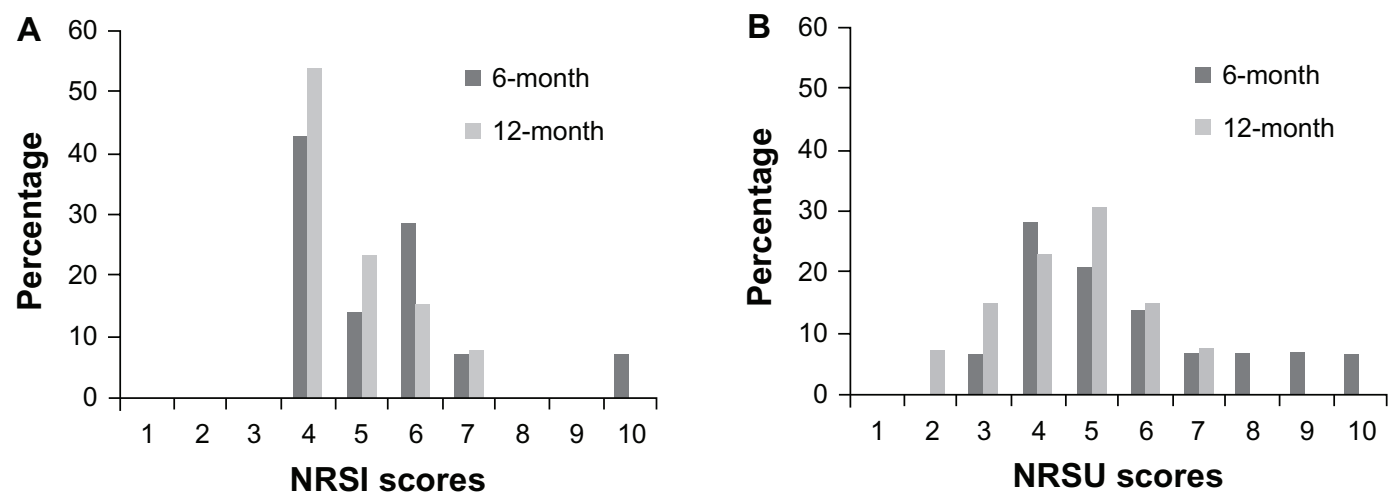

Figure 2 Pain intensity (A) and pain unpleasantness (B) scores 6 and 12 months after surgery for children with moderate/severe chronic postsurgical pain. Abbreviations: NRSI, Numeric Rating Scale for pain intensity; NRSU, Numeric Rating Scale for pain unpleasantness.

\section{Risk factors for the transition from APSP to CPSP \\ Risk factors (48-72 hours) for 6-month moderate/severe CPSP}

Results of the logistic regression analysis showed that pain unpleasantness $(B=0.272, P=0.046)$ was the only significant predictor of 6-month chronic pain status $\left(\chi^{2}=4.34\right.$, $P=0.037)$.

\section{Risk factors (48-72 hours) for I 2-month} moderate/severe CPSP

Results of the logistic regression analysis showed that not one patient-related or psychological factor predicted the presence of moderate/severe CPSP 12 months after surgery.

\section{Risk factors for the maintenance of moderate/severe CPSP \\ Six-month risk factors for 12-month moderate/severe CPSP}

Results of the logistic regression analysis showed that anxiety sensitivity ( $B=0.138, P=0.043$ ) was the only significant predictor of the maintenance of or recovery from moderate/severe CPSP 12 months after surgery $\left(\chi^{2}=5.02\right.$, $P=0.025)$.

\section{Discussion}

The present study was designed to examine the course of pediatric moderate/severe CPSP and model the development and maintenance of moderate/severe CPSP from the acute postsurgical period.

Table 3 Differences in CPSP and functional disability trajectories in children with moderate to severe CPSP or no/mild CPSP at 12 months (CPSP status) using generalized estimating equation

\begin{tabular}{|c|c|c|c|c|c|}
\hline & Wald Chi-square & df & $P$-value & B & Standard error \\
\hline \multicolumn{6}{|l|}{ Pain-intensity trajectory } \\
\hline Intercept & 212.3 & 1 & $<0.001$ & I.I & 0.1 \\
\hline Time & 0.9 & 1 & 0.338 & 0.03 & 0.03 \\
\hline Asymptotic time & 29.8 & I & $<0.001$ & 2.8 & 0.5 \\
\hline CPSP status & 2.3 & 1 & 0.127 & 0.2 & 0.2 \\
\hline CPSP status* asymptotic time & 38.9 & 1 & $<0.001$ & -2.4 & 0.4 \\
\hline \multicolumn{6}{|c|}{ Pain-unpleasantness trajectory } \\
\hline Intercept & 211.9 & I & $<0.001$ & 1.3 & 0.1 \\
\hline Time & 2.7 & I & 0.100 & 0.1 & 0.03 \\
\hline Asymptotic time & 28.3 & 1 & $<0.001$ & 3.2 & 0.6 \\
\hline CPSP status & 5.3 & I & 0.021 & 0.4 & 0.2 \\
\hline CPSP status* asymptotic time & 21.4 & I & $<0.001$ & -2.1 & 0.4 \\
\hline
\end{tabular}

Notes: Corrected quasi-likelihood under independence-model criterion for pain-intensity trajectory = 399.3; corrected quasi-likelihood under independence-model criterion for pain-unpleasantness trajectory $=504.5$.

Abbreviations: CPSP, chronic postsurgical pain; df, degrees of freedom. 

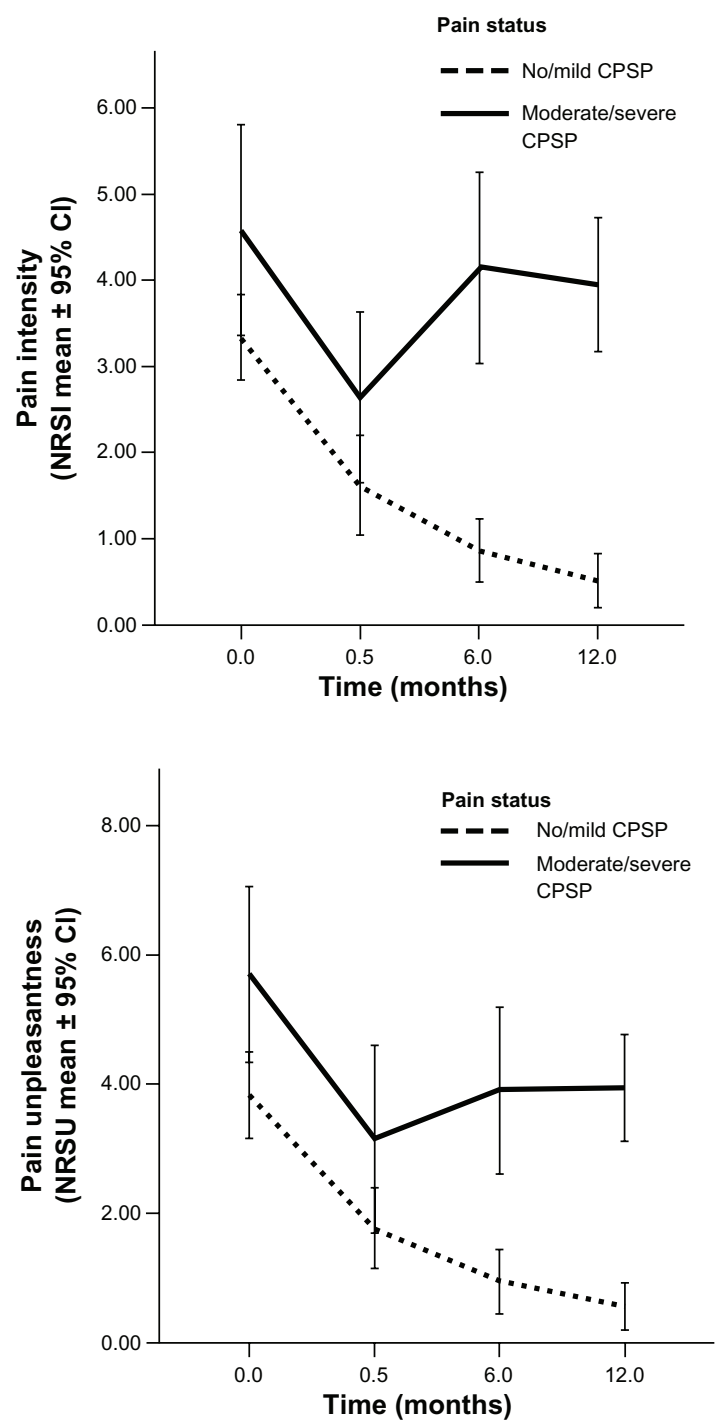

Figure 3 Differences in pain intensity and unpleasantness trajectories between children with moderate/severe CPSP and without or with mild CPSP. Note: Error bars represent the $95 \%$ confidence intervals.

Abbreviations: CPSP, chronic postsurgical pain; NRSI, Numeric Rating Scale for pain intensity; NRSU, Numeric Rating Scale for pain unpleasantness; $\mathrm{Cl}$, confidence interval.

\section{Description of pediatric moderate/severe CPSP and modeling of pain intensity and unpleasantness trajectories}

The results of the present study show that almost one-quarter of children who underwent major surgery developed CPSP of moderate to severe intensity and unpleasantness 1 year after surgery. About one-third of children with CPSP 6 months after surgery reported experiencing pain daily. Approximately $14 \%$ of children with CPSP reported severe (NRS $\geq 7$ out of 10 ) levels of pain 6 months after surgery $(n=2)$. These statistics are similar to those found in adults; a review of CPSP suggests that $10 \%$ of patients report severe, intractable CPSP. ${ }^{58} \mathrm{~A}$ recent retrospective study of pediatric CPSP found that $13 \%$ of children between 3 and 18 months following surgery report current pain at the surgery site. ${ }^{6}$ The 12 -month incidence of moderate/severe CPSP in the present study was higher (22\%). This difference in prevalence estimates could be due to the type of surgical procedures studied; children in the present study all underwent major orthopedic or general surgery, and as such the magnitude of tissue damage might contribute to the higher prevalence rate found.

In general, pediatric chronic pain is prevalent but typically not accompanied by severe levels of disability. ${ }^{59}$ Approximately $5 \%-15 \%$ of children with chronic pain report pain disability of a severity requiring professional attention. ${ }^{59,60}$ Results from the present study are consistent with this literature. The presence of moderate/severe CPSP was not accompanied by high levels of functional disability for the majority of children or with the use of pain medication in the first year following surgery. The absence of clinically relevant levels of functional disability might be due in part to the use of a measure of general disability and not one related specifically to pain. Participants are asked to rate items of the FDI based on the level of difficulty they had in completing specific tasks; it does not specify that these difficulties must have been related to the pain they were experiencing. As such, a participant might report higher levels of disability because of a cold/flu, for example. Alternatively, it could be that because of the way children cope with everyday problems, including pain, ${ }^{61}$ they do not experience much interference from the pain. Children often have little experience coping with chronic pain on their own and instead rely on parental involvement and support to cope and function. ${ }^{61}$ Consequently, the low levels of functional disability in the present study may indicate that parents pain are providing the necessary support so that even children with moderate to severe pain are protected from the interfering effects of pain.

Results indicate that while there is little difference in pain-intensity scores 48-72 hours after surgery between children who developed moderate/severe CPSP vs no/ mild CPSP, the former reported significantly higher pain intensity and unpleasantness levels 2 weeks after discharge from hospital compared to the latter. Pain intensity and unpleasantness scores 2 weeks after discharge from hospital provided valuable information about the risk of developing moderate/severe CPSP. Children who reported pain intensity and/or pain unpleasantness scores of 3 or higher on the NRS two weeks after discharge were more than three times as likely to report moderate/severe CPSP 6 months later and more than twice as likely to report moderate/severe CPSP 12 months later than children who reported NRS scores lower than 3 . We do not know whether intense acute pain is a causal risk 
Table 4 Relative risk of developing moderate/severe CPSP based on 2-week postdischarge pain intensity and unpleasantness scores

\begin{tabular}{|c|c|c|c|c|c|}
\hline & \multirow{2}{*}{$\begin{array}{l}\text { NRSI score } 2 \text { weeks } \\
\text { after discharge }\end{array}$} & \multicolumn{2}{|l|}{ Pain status (n) } & \multirow[t]{2}{*}{ Relative risk } & \multirow{2}{*}{$\begin{array}{l}95 \% \text { confidence } \\
\text { interval }\end{array}$} \\
\hline & & $\begin{array}{l}\text { Moderate/severe } \\
\text { CPSP }\end{array}$ & No/mild CPSP & & \\
\hline \multicolumn{6}{|l|}{ Pain intensity } \\
\hline 6 months after & NRSI $\geq 4$ & 6 & 8 & 3.3 & $1.2-9.0$ \\
\hline surgery & NRSI $<4$ & 5 & 33 & 0.7 & $0.4-1.1$ \\
\hline 12 months after & $N R S I \geq 4$ & 5 & 10 & 2.5 & $0.9-7.5$ \\
\hline surgery & NRSI $<4$ & 5 & 33 & 0.8 & $0.5-1.1$ \\
\hline \multicolumn{6}{|c|}{ Pain unpleasantness } \\
\hline 6 months after & NRSU $\geq 4$ & 6 & 11 & 2.5 & $0.9-7.0$ \\
\hline surgery & NRSU $<4$ & 5 & 30 & 0.8 & $0.5-1.1$ \\
\hline 12 months after & NRSU $\geq 4$ & 6 & 13 & 2.7 & $0.6-8.3$ \\
\hline surgery & NRSU $<4$ & 4 & 30 & 0.8 & $0.6-1.1$ \\
\hline
\end{tabular}

Abbreviations: CPSP, chronic postsurgical pain; NRSI, Numeric Rating Scale for pain intensity; NRSU, Numeric Rating Scale for pain unpleasantness.

factor for CPSP, but the present results raise the possibility that intervening with children and adolescents who present with NRSI scores greater than 3 out of 10 two weeks after major surgery might help prevent the transition from acute to CPSP. Future research might consider looking at whether pharmacological, psychological, social, or a combination of interventions in the acute postoperative period successfully prevent the development of CPSP or reduce the incidence and/or intensity of CPSP.

\section{Risk factors for the transition from APSP to CPSP and maintenance of moderate/ severe CPSP}

Factors that predict the transition to chronicity differ from those that predict the maintenance or persistence of adult chronic pain. ${ }^{32,33}$ Although research has not yet examined CPSP in children, studies of pediatric widespread pain suggests that factors predicting the onset of widespread pain (conduct and emotional problems and other somatic symptoms) differ somewhat from those that predict the maintenance of pain (behavioral characteristics and somatic symptoms). ${ }^{34}$ The present results are consistent with these findings. Anxiety sensitivity emerged as a significant predictor of moderate/ severe CPSP between 6 months and 1 year after surgery (but not earlier), and as such might be an important marker that maintains CPSP once it has developed. In contrast, pain unpleasantness appears to be involved in the transition from APSP to CPSP but interestingly not in its maintenance.

Anxiety sensitivity has been found to play an important role in the pain experience of individuals due to the negative interpretations of pain-related sensations. ${ }^{62} \mathrm{~A}$ recent metaanalytic review of anxiety sensitivity in clinical and nonclinical pain populations found that anxiety sensitivity is strongly associated with fear of pain, which can lead to avoidance behaviors and the maintenance of chronic pain; as such, fear of pain has been suggested as a bridge to explain the association between anxiety sensitivity and pain (see Ocanez et $\mathrm{al}^{62}$ for a review). Consistent with the diathesis-stress model of chronic pain and disability ${ }^{28,63}$ and the fear-avoidance models of adult ${ }^{64}$ and child ${ }^{65}$ chronic pain, by amplifying pain-related fears, anxiety sensitivity is associated with avoidance behaviors and ultimately the maintenance of chronic pain. ${ }^{64}$ Results from the present study are consistent with the theoretical models and supportive empirical evidence suggesting that this construct may play an important role in the maintenance of the pediatric chronic pain experience. ${ }^{65}$ It has yet to be determined if fear of pain is a mediator in the observed relationship between anxiety sensitivity and the maintenance of pediatric moderate/severe CPSP.

Results from the present study contrast with those found in the adult literature suggesting that patient-related factors (particularly APSP intensity) predict the development of CPSP. ${ }^{66}$ Pain unpleasantness, but not pain intensity, was found to predict the development of moderate/severe CPSP. These results suggest that the affective dimension of the pain experience might play a particularly important role in the development of pediatric chronic pain.

These results are not consistent either with adult studies demonstrating the important role of psychological factors (particularly pain catastrophizing) in predicting the development of CPSP. ${ }^{66}$ Pain catastrophizing is also an important component of the pediatric fear-avoidance model of chronic pain and has received empirical support for its role in pediatric chronic pain and disability. ${ }^{65}$ Researchers have recently questioned the content validity of the paincatastrophizing construct for use in children, in part because it lacks developmental and social contextualization (eg, parental psychological factors might have a direct impact on 
children's response to pain $^{65}$ ) in this patient population and because it fails to capture social and cognitive milestones associated with youth development. ${ }^{61}$ Additional research is needed to examine the role of pain catastrophizing in the development of CPSP within a social, cognitive, and developmental context.

Pain anxiety did not emerge as a significant predictor of the development or maintenance of CPSP. It is possible that pain anxiety, while not directly predicting the development or maintenance of chronic pain, is indirectly related to pain outcomes through its influence on certain pain behaviors, such as escape and avoidance behaviors. Future research should examine the role of pain anxiety as a potential moderator of the relationship between pain behaviors and pain outcomes.

\section{Limitations}

All measures were collected postoperatively, and as such preoperative baseline data were not collected regarding the participants' pain-related psychological distress. Second, children recruited in this study all underwent a major surgical procedure, and as such results cannot be generalized to children who are undergoing less invasive surgery. Third, the response rate for this study was $55 \%$. It is possible that children with higher APSP or higher postoperative anxiety refused to participate. Fourth, 25\% of participants did not complete the 6- and 12-month follow-ups, and consequently analyses of CPSP are based on $75 \%$ of the sample. Fifth, this study focused on psychological risk factors for the development of CPSP and did not consider biological factors that can influence the development and/or maintenance of CPSP. It is possible that, for example, the type of analgesic regimen administered perioperatively influenced the development of CPSP through central sensitization. Additional research is needed to understand the biopsychosocial factors involved in the development and maintenance of CPSP. Finally, our use of a 4-point FDI scale instead of the typical; 5-point scale may have led to inaccurate levels of functional disability.

\section{Conclusion}

In conclusion, this study is the first to prospectively examine the development and persistence of pediatric CPSP. Results suggest that moderate/severe CPSP is a frequent occurrence, despite low levels of associated functional disability. Importantly, a cutoff score of 3.0 on the NRS for pain intensity or pain unpleasantness 2 weeks after discharge is associated with more than twice the risk of developing moderate/ severe CPSP 1 year after surgery. The factors that predict the development of moderate/severe CPSP appear to differ from those that predict its persistence. Future studies with additional time points would help to differentiate risk factors associated with the development and maintenance of moderate/severe CPSP. Pain unpleasantness is involved in the development of moderate/severe CPSP, while anxiety sensitivity appears to play a significant role in its persistence. This line of research has the potential to provide valuable information for the development of preventive interventions that can reduce the occurrence of moderate/severe CPSP in this patient population.

\section{Acknowledgments}

MGP is supported by a Canada Graduate Scholarship Doctoral Award from the Canadian Institutes of Health Research (CIHR). MGP is a recipient of a LillianWright Maternal-Child Health Scholarship from York University, a trainee member of Pain in Child Health and a CIHR Strategic Training Fellow in Pain: Molecules to Community. JS is supported by a Ministry of Health and Long-Term Care Career Scientist Award. JK is supported by a CIHR Canada Research Chair in Health Psychology at York University.

\section{Disclosure}

The authors have no conflicts of interest.

\section{References}

1. Macrae WA, Davies HTO. Chronic postsurgical pain. In: Crombie IK, Linton S, Croft P, Von Knorff M, LeResche L, editors. Epidemiology of Pain. Seattle: IASP; 1999:125-142.

2. Katz J, Pagé MG. Identification of risk and protective factors in the transition from acute to chronic post surgical pain. In: Lynch ME, Craig KD, Peng PWH, editors. Clinical Pain Management: A Practical Guide. Oxford, UK: Wiley-Blackwell; 2010:32-41.

3. Crombie IK, Davies HT, Macrae WA. Cut and thrust: antecedent surgery and trauma among patients attending a chronic pain clinic. Pain. 1998;76(1-2):167-171.

4. Wong GT, Yuen VM, Chow BF, Irwin MG. Persistent pain in patients following scoliosis surgery. Eur Spine J. 2007;16(10):1551-1556.

5. Aasvang EK, Kehlet H. Chronic pain after childhood groin hernia repair. J Pediatr Surg. 2007;42(8):1403-1408.

6. Fortier MA, Chou J, Maurer EL, Kain ZN. Acute to chronic postoperative pain in children: preliminary findings. J Pediatr Surg. 2011;46(9): $1700-1705$.

7. Kristensen AD, Pedersen TA, Hjortdal VE, Jensen TS, Nikolajsen L. Chronic pain in adults after thoracotomy in childhood or youth. $\mathrm{Br} J$ Anaesth. 2010;104(1):75-79.

8. Hanley MA, Jensen MP, Ehde DM, Hoffman AJ, Patterson DR, Robinson LR. Psychosocial predictors of long-term adjustment to lower-limb amputation and phantom limb pain. Disabil Rehabil. 2004;26(14-15):882-893.

9. Hanley MA, Jensen MP, Smith DG, Ehde DM, Edwards WT, Robinson LR. Preamputation pain and acute pain predict chronic pain after lower extremity amputation. J Pain. 2007;8(2):102-109.

10. Jensen MP, Ehde DM, Hoffman AJ, Patterson DR, Czerniecki JM, Robinson LR. Cognitions, coping and social environment predict adjustment to phantom limb pain. Pain. 2002;95(1-2):133-142. 
11. Katz J, Selter Z. Transition from acute to chronic postsurgical pain: risk factors and protective factors. Expert Rev Neurother. 2009;9(5): 723-744

12. Bruce J, Quinlan J. Chronic post surgical pain. Rev Pain. 2011;5(23):23-29.

13. Niraj G, Rowbotham DJ. Persistent postoperative pain: where are we now? Br J Anaesth. 2011;107(1):25-29.

14. Pavlin DJ, Sullivan MJ, Freund PR, Roesen K. Catastrophizing: a risk factor for postsurgical pain. Clin J Pain. 2005;21(1):83-90.

15. Voscopoulos C, Lema M. When does acute pain become chronic? $\mathrm{Br}$ J Anaesth. 2010;105 Suppl 1:i69-i85.

16. Hinrichs-Rocker A, Schulz K, Järvinen I, Lefering R, Simanski C, Neugebauer EA. Psychosocial predictors and correlates for chronic post-surgical pain (CPSP) - a systematic review. Eur J Pain. 2009;13(7):719-730.

17. Ahn JC, Fortier MA, Kain ZN. Acute to chronic postoperative pain in children: does it exists? Pain Manage. 2012;2(5):421-423.

18. McCracken LM, Dhingra L. A short version of the Pain Anxiety Symptoms Scale (PASS-20): preliminary development and validity. Pain Res Manage. 2002;7(1):45-50.

19. McCracken LM, Zayfert C, Gross RT. The Pain Anxiety Symptoms Scale: development and validation of a scale to measure fear of pain Pain. 1992;50(1):67-73.

20. Crombez G, Eccleston C, Baeyens F, Eelen P. When somatic information threatens, catastrophic thinking enhances attentional interference. Pain. 1998;75(2-3):187-198.

21. Sullivan MJL, Bishop SR, Pivik J. The pain catastrophizing scale: development and validation. Psychol Assess. 1995;7(4):524-532.

22. Reiss S, McNally RJ. Expectancy model of fear. In: Reiss S, Bootzin RR, editors. Theoretical Issues in Behavior Therapy. San Diego: Academic Press; 1985:107-121.

23. Martin AL, McGrath PA, Brown SC, Katz J. Anxiety sensitivity, fear of pain and pain-related disability in children and adolescents with chronic pain. Pain Res Manage. 2007;12(4):267-272.

24. Vervoort T, Goubert L, Eccleston C, Bijttebier P, Crombez G Catastrophic thinking about pain is independently associated with pain severity, disability, and somatic complaints in school children and children with chronic pain. J Pediatr Psychol. 2006;31(7):674-683.

25. Tsao JC, Lu Q, Myers CD, Kim SC, Turk N, Zeltzer LK. Parent and child anxiety sensitivity: relationship to children's experimental pain responsivity. J Pain. 2006;7(5):319-326.

26. Tsao JC, Meldrum M, Kim SC, Zeltzer LK. Anxiety sensitivity and health-related quality of life in children with chronic pain. J Pain. 2007;8(10):814-823

27. Tsao JC, Myers CD, Craske MG, Bursch B, Kim SC, Zeltzer LK Role of anticipatory anxiety and anxiety sensitivity in children's and adolescents' laboratory pain responses. J Pediatr Psychol. 2004; 29(5):379-388.

28. Turk DC. A diathesis-stress model of chronic pain and disability following traumatic injury. Pain Res Manag. 2002;7(1):9-19.

29. Lethem J, Slade PD, Troup JD, Bentley G. Outline of a fearavoidance model of exaggerated pain perception - I. Behav Res Ther. 1983;21(4):401-408.

30. Vlaeyen JWS, Kole-Snijders AM, Boeren RG, van Eek H. Fear of movement/(re)injury in chronic low back pain and its relation to behavioral performance. Pain. 1995;62(3):363-372

31. Vlaeyen JWS, Linton S. Fear-avoidance and its consequences in chronic musculoskeletal pain: a state of the art. Pain. 2000;85(3):317-332.

32. Katz J, Asmundson GJ, McRae K, Halket E. Emotional numbing and pain intensity predict the development of pain disability up to one year after lateral thoracotomy. Eur J Pain. 2009;13(8):870-878.

33. Katz J. One man's risk factor is another man's outcome: difference in risk factor profiles for chronic postsurgical pain maintenance vs transition. Pain. 2012;153(3):505-506.

34. Jones GT, Silman AJ, Macfarlane GJ. Predicting the onset of widespread body pain among children. Arthritis Rheum. 2003;48(9):2615-2621.

35. Kehlet H, Rathmell JP. Persistent postsurgical pain: the path forward through better design of clinical studies. Anesthesiology. 2010;112(3): 514-515.
36. Gerbershagen HJ, Rothaug J, Kalkman CJ, Meissner W. Determination of moderate-to-severe postoperative pain on the numeric rating scale: a cut-off point analysis applying four different methods. Br J Anaesth. 2011;107(4):619-626.

37. Jensen MP, Smith DG, Ehde DM, Robinsin LR. Pain site and the effects of amputation pain: further clarification of the meaning of mild, moderate, and severe pain. Pain. 2001;91(3):317-322.

38. Landman Z, Oswald T, Sanders J, Diab M. Prevalence and predictors of pain in surgical treatment of adolescent idiopathic scoliosis. Spine (Phila Pa 1976). 2011;36(10):825-829.

39. Pagé MG, Fuss S, Martin AL, Escobar EM, Katz J. Development and preliminary validation of the Child Pain Anxiety Symptoms Scale in a community sample. J Pediatr Psychol. 2010;35(10):1071-1082.

40. McCracken LM, Dhingra L. A short version of the Pain Anxiety Symptoms Scale (PASS-20): preliminary development and validity. Pain Res Manag. 2002;7(1):45-50.

41. Page MG, Campbell F, Isaac L, Stinson J, Martin-Pichora AL, Katz J. Reliability and validity of the Child Pain Anxiety Symptoms Scale (CPASS) in a clinical sample of children and adolescents with acute postsurgical pain. Pain. 2011;152(9):1958-1965.

42. Silverman WK, Fleisig W, Rabian B, Peterson RA. Childhood Anxiety Sensitivity Scale. J Clin Child Psychol. 1991;20(2):162-168.

43. Crombez G, Bijttebier P, Eccleston C, et al. The child version of the pain catastrophization scale (PCS-C): a preliminary validation. Pain. 2003;104(3):639-646

44. Sullivan MJL, Bishop SR, Pivik J. The pain catastrophizing scale: development and validation. Psychol Assess. 1995;7(4):524-532.

45. Crombez G, Eccleston C, Baeyens F, Eelen P. When somatic information threatens, pain catastrophizing enhances attentional interference. Pain. 1998;75(2-3):187-198.

46. Walker LS, Greene JW. The functional disability inventory: measuring a neglected dimension of child health status. J Pediatr Psychol. 1991; 16(1):39-58.

47. Kashikar-Zuck S, Vaught MH, Goldschneider KR, Graham TB, Miller JC. Depression, coping, and functional disability in juvenile fibromyalgia syndrome. J Pain. 2002;3(5):412-419.

48. Lynch AM, Kashikar-Zuck S, Goldschneider KR, Jones BA. Psychosocial risks for disability in children with chronic back pain. J Pain. 2006;7(4):244-251.

49. Reid GJ, McGrath PJ, Lang BA. Parent-child interactions among children with juvenile fibromyalgia, arthritis and healthy controls. Pain. 2005; 113(1-2):201-210.

50. Gidron Y, McGrath PJ, Goodday R. The physical and psychosocial predictors of adolescents' recovery from oral surgery. J Behav Med. 1995; 18(4):385-399.

51. Von Baeyer CL. Numerical rating scale for self-report of pain intensity in children and adolescents: recent progress and further questions. Eur J Pain. 2009;13(10):1005-1007.

52. von Baeyer CL, Spagrud LJ, McCormick JC, Choo E, Neville K, Connelly MA. Three new datasets supporting use of the Numerical Rating Scale (NRS-11) for children's self-reports of pain intensity. Pain. 2009;143(3):223-227.

53. Faulstich ME, Carey MP, Ruggiero L, Enyart P, Gresham F. Assessment of depression in childhood and adolescence: an evaluation of the Center for Epidemiological Studies Depression Scale for Children (CES-DC). Am J Psychiatry. 1986;143(8):1024-1027.

54. March JS, Parker JDA, Sullivan K, Stallings P, Conners CK. The Multidimensional Anxiety Scale for Children (MASC): factor structure, reliability and validity. J Am Acad Child Adolesc Psychiatry. 1997;36(4):554-565.

55. Faul F, Erdfelder E, Buchner A, Lang AG. Statistical power analyses using $\mathrm{G}^{*}$ Power 3.1: tests for correlation and regression analyses. Behav Res Methods. 2009;41(4):1149-1160.

56. Gelman A, Hill J. Sample size and power calculations. In: Gelman A, Hill J. Data Analysis Using Regression and Multilevel/ Hierarchical Models. New York: Cambridge University Press; 2007: $437-455$. 
57. Hsieh FY, Bloch DA, Larsen MD. A simple method of sample size calculation for linear and logistic regression. Stat Med. 1998;17(14): 1623-1634.

58. Katz J, Seltzer Z. Transition from acute to chronic postsurgical pain: risk factors and protective factors. Expert Rev Neurother. 2009; 9(5):723-744.

59. von Baeyer CL. Interpreting the high prevalence of pediatric chronic pain revealed in community surveys. Pain. 2011;152(12): 2683-2684.

60. Huguet A, Miro J. The severity of chronic pediatric pain: an epidemiological study. J Pain. 2008;9(3):226-236.

61. Eccleston C, Fisher EA, Vervoort T, Crombez G. Worry and catastrophizing about pain in youth: a reappraisal. Pain. 2012;153(8): 1560-1562.

62. Ocanez KL, McHugh RK, Otto MW. A meta-analytic review of the association between anxiety sensitivity and pain. Depress Anxiety. 2010;27(8):760-767.
63. Martin AL, Halket E, Asmundson GJ, Flora DB, Katz J. Posttraumatic stress symptoms and the diathesis-stress model of chronic pain and disability. Clin J Pain. 2010;26(6):518-527.

64. Asmundson GJ, Norton PJ, Vlaeyen JWS. Fear-avoidance models of chronic pain: an overview. In: Asmundson GJG, Vlaeyen JWS, Crombez G, editors. Understanding and Treating Fear of Pain. New York: Oxford University Press; 2004:3-24.

65. Asmundson GJ, Noel M, Petter M, Parkerson H. Pediatric fearavoidance model of chronic pain: Foundation, application and future directions. Pain Res Manag. 2012;17(6):397-405.

66. Katz J, Seltzer Z. Transition from acute to chronic postsurgical pain: risk factors and protective factors. Expert Rev Neurother. 2009;9(5): 723-744.

\section{Publish your work in this journal}

The Journal of Pain Research is an international, peer-reviewed, open access, online journal that welcomes laboratory and clinical findings in the fields of pain research and the prevention and management of pain. Original research, reviews, symposium reports, hypothesis formation and commentaries are all considered for publication.

\section{Dovepress}

The manuscript management system is completely online and includes a very quick and fair peer-review system, which is all easy to use. Visit http://www.dovepress.com/testimonials.php to read real quotes from published authors. 